\title{
Triple negative breast cancer: clinicopathological characteristics and treatment strategies
}

\author{
Futoshi Akiyama · Hirotaka Iwase
}

Published online: 9 June 2009

(C) The Japanese Breast Cancer Society 2009

\begin{abstract}
Triple negative breast cancer (TNBC) is defined as a subtype that is negative for estrogen receptor, progesterone receptor and human epidermal growth factor receptor 2. We introduce the theme of the special issue concerning clinicopathological characteristics and prospective treatment strategies of TNBC. This special issue consists of five conference papers that have been agreed on by the speakers and the commentator of the symposium of the 16th Annual Meeting of the Japanese Breast Cancer Society in September 2008 in Osaka.
\end{abstract}

Keywords Triple negative breast cancer .

Basal-like tumor

Triple negative breast cancer (TNBC) is a clinically relevant term referring to breast carcinomas that do not express the estrogen receptor (ER), progesterone receptor (PgR) and human epidermal growth factor receptor type 2 (HER2). This subtype has been considered to be a clinicopathological entity with aggressive behaviors and poor prognosis because of the absence of a specific target for the tumor, such as in endocrine therapy and anti-HER2 therapy, although these tumors with TNBC are sensitive to

\footnotetext{
F. Akiyama

Department of Pathology, Cancer Institute, 3-10-6 Ariake,

Koto-ku, Tokyo 135-8550, Japan

e-mail: fakiyama@jfcr.or.jp

H. Iwase $(\square)$

Department of Breast and Endocrine Surgery,

Faculty of Medical and Pharmaceutical Sciences,

Kumamoto University, Honjo 1-1-1,

Kumamoto 860-8556, Japan

e-mail: hiwase@kumamoto-u.ac.jp
}

chemotherapy $[1,2]$. TNBC is not a rare subtype of invasive ductal carcinoma of the breast. Of 72,694 cases registered by the Registration Committee of the Japanese Breast Cancer Society (JBCS) from January 2004 to January 2009, 63,296 (87.1\%) were examined for ER, PgR and HER2. Of these, the TN subtype was seen in 9,094 cases (14.4\%), and the luminal type, such as with ER- and/or PgR-positive and HER2-negative subtypes, was seen in $70.0 \%$ (Fig. 1).

Firstly, Dr. Sasaki and Dr. Tsuda discuss the clinicopathological characteristics of TNBC, especially the relationship among the TNBC and DNA microarray subtypes, basal-like subtype and normal breast (or unclassified) subtype, the former being correlated with an aggressive clinical course. The basal-like subtype is characterized by expression of myoepithelial/basal markers, such as cytokeratin (CK) 5/6 and CK14, and epidermal growth factor receptor (EGFR). Furthermore, they show the similarities of basal-like tumors to carcinomas with BRCA1 deficiency and the biological features.

Secondly, Dr. Yamamoto and his colleagues report their original work entitled "Clinical significance of basal-like subtype in TNBC." They investigate the prognosis of TNBC using immunohistochemical techniques, such as CK5/6, EGFR, c-kit, Ki-67 and others. When the basal-like type is defined as CK5/6 positive and/or EGFR positive in TNBC, the basal-like tumor has a poor prognosis, high nuclear grade, high Ki-67 labeling index and c-kit expression. They conclude that basal-like tumors should be divided into at least two types of cancer concerning a prognostic value. Thirdly, Dr. Ohta and his colleagues focus on the deficiency of BRCA1 function and DNA double-strand break repair in basal-like tumors based on their own research works. They also reach target therapies with respect to increasing the sensitivity of cells to DNA 


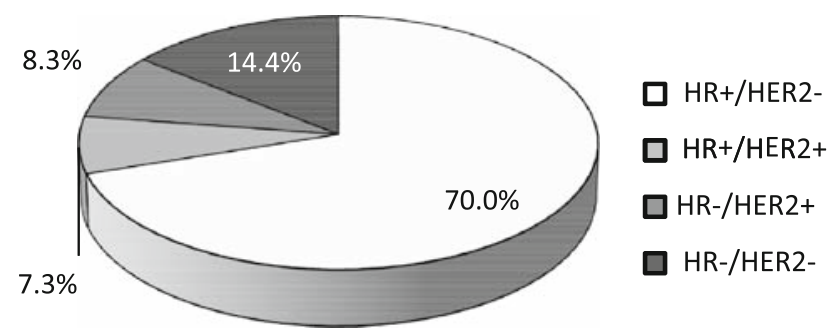

HR+: ER positive and/or PgR positive HR-: ER negative and PgR negative

Fig. 1 Receptor subtype in Japanese breast cancer patients $(n=63,296 ;$ JBCS, 2004/1/1-2009/1/7)

damage-inducing agents, such as PARP inhibitors, DNA cross-linkers or topoisomerase inhibitors.

Fourthly, Dr. Kurebayashi reviews the treatment strategies for TNBC on the basis of molecular characteristics. Novel target agents, such as poly ADP-ribose polymerase (PARP) inhibitors, cetuximab; anti-EGFR antibody, erlotinib; EGFR tyrosine kinase inhibitor, imatinib, sunitinib, dasatinib; Src/c-kit inhibitors and everolimus; and mTOR inhibitors, are reported in preclinical and clinical settings. Especially, PARP inhibitor is thought to be most promising in the treatment of TNBC. Finally, Dr. Sakamoto and Dr. Honma report their own work concerning the relationship of $\operatorname{ER} \beta 1$ expression in TNBC [3] and apocrine carcinoma of the breast, which often fall into the category of TNBC. $\mathrm{ER} \beta 1$ might be one of the distinct markers to divide basal and non-basal tumors.

Taken together, to understand the biological characteristics of TNBC and to optimize several novel target treatments for the patients with TNBC, large, systemic and prospective translational research linked to clinical trials is warranted.

Acknowledgment We wish to thank Mr. Naohito Fukui, NPO Japan Clinical Research Support Unit, and Dr. Takuji Iwase, Director of the Registration Committee of the JBCS, for offering the registration data from JBCS.

\section{References}

1. Iwase $\mathrm{H}$, Yamamoto $\mathrm{Y}$. Biological characteristics of triple negative breast cancer. Jpn J Breast Cancer. 2008;23(2):75-80.

2. Kobayashi S. Basal-like subtype of breast cancer: a review of its unique characteristics and their clinical significance. javascript:AL_get(this, 'jour', 'Breast Cancer.'). Breast Cancer. 2008; 15(2):153-8.

3. Honma N, Horii R, Iwase T, Saji S, Younes M, Takubo K, et al. Clinical importance of estrogen receptor-b evaluation in breast cancer patients treated with adjuvant tamoxifen therapy. J Clin Oncol. 2008;26:3727-34. 